\title{
Orientation-Preservation Conditions on an Iso-parametric FEM in Cavitation Computation*
}

\author{
Chunmei Su, Zhiping $\mathrm{Li}^{\dagger}$ \\ LMAM \& School of Mathematical Sciences, \\ Peking University, Beijing 100871, China
}

\begin{abstract}
The orientation-preservation condition, i.e., the Jacobian determinant of the deformation gradient det $\nabla u$ is required to be positive, is a natural physical constraint in elasticity as well as in many other fields. It is well known that the constraint can often cause serious difficulties in both theoretical analysis and numerical computation, especially when the material is subject to large deformations. In this paper, we derive a set of sufficient and necessary conditions for the quadratic iso-parametric finite element interpolation functions of cavity solutions to be orientation preserving on a class of radially symmetric large expansion accommodating triangulations. The result provides a practical quantitative guide for meshing in the neighborhood of a cavity and shows that the orientation-preservation can be achieved with a reasonable number of total degrees of freedom by the quadratic isoparametric finite element method.
\end{abstract}

Keywords: orientation-preservation condition, iso-parametric FEM, cavitation computation, nonlinear elasticity

\footnotetext{
*The research was supported by the NSFC projects 11171008 and 11571022.

${ }^{\dagger}$ Corresponding author, email: lizp@math.pku.edu.cn
} 


\section{Introduction}

As early as 1958, Gent and Lindley [6] carried out physical experiments and studied the sudden void formation on elastic bodies under hydrostatic tension. Since then, the phenomenon, which is referred to as cavitation in literatures, has been intensively studied by numerous researchers.

There are two representative models for the cavity formation. One is the socalled deficiency model proposed by Gent and Lindley [6], in which the cavities are considered to develop from pre-existing small voids under large triaxial tensions. The other is the perfect model established by Ball [2], in which voids form in an intact body so that the total stored energy of the material could be minimized. The relations between the two models are partially established by the work of Sivaloganathan et. al. [18] and Henao [7]: roughly speaking, given the right positions of the voids, as the radii of the pre-existing small voids go to zero, the solution of the deficiency model converges to the solution of the perfect model. Furthermore, the configurational forces can be used to detect whether a void is formed in the right position $[11,20]$.

The perfect model typically displays the Lavrentiev phenomenon [8] when there is a cavitation solution, leading to the failure of the conventional finite element methods $[1,4]$. Though there are existing numerical methods developed to overcome the Lavrentiev phenomenon $([1,4,9,14])$, they do not seem to be powerful and efficient enough to tackle the cavitation problem on their own.

In fact, all of the numerical studies on cavitation, known to the authors so far, are based on the deficiency model, in which one considers to minimize the total energy of the form

$$
E(u)=\int_{\Omega_{\varrho}} W(\nabla u(x)) d x,
$$

in the set of admissible functions

$$
U=\left\{u \in W^{1,1}\left(\Omega_{\varrho} ; \mathbb{R}^{n}\right) \text { is one-to-one a.e. : }\left.u\right|_{\Gamma_{0}}=u_{0} \text {, } \operatorname{det} \nabla u>0 \text { a.e. }\right\},
$$

where $\Omega_{\varrho}=\Omega \backslash \bigcup_{i=1}^{K} B_{\varrho_{i}}\left(a_{i}\right) \subset \mathbb{R}^{n}(n=2,3)$ denotes the region occupied by an 
elastic body in its reference configuration, $B_{\varrho_{i}}\left(a_{i}\right)=\left\{x \in \mathbb{R}^{n}:\left|x-a_{i}\right|<\varrho_{i}\right\}$ are the pre-existing defects of radii $\varrho_{i}$ centered at $a_{i}$. In (1.1) $W: M_{+}^{n \times n} \rightarrow \mathbb{R}^{+}$ is the stored energy density function of the material, $M_{+}^{n \times n}$ denotes the set of $n \times n$ matrices with positive determinant, $\Gamma_{0}$ is the boundary of $\Omega$. We notice here that, in elasticity theory, the Jacobian determinant $\operatorname{det} \nabla u$, the local volume "stretching factor" of a deformation, is naturally required to be positive, which means that no volume of the material is compressed into a point or even turned "inside out". The constraint is of vital importance, for instance it excludes the deformations that have a reflection component, and it is a necessary condition of the fact that the matter should not inter-penetrate. On the other hand, the constraint $\operatorname{det} \nabla u>0$, though less strict than the incompressibility $\operatorname{det} \nabla u \equiv 1$, also inevitably brings some serious difficulties to mathematical models $([3,16])$ as well as numerical computations $([5,11,25])$.

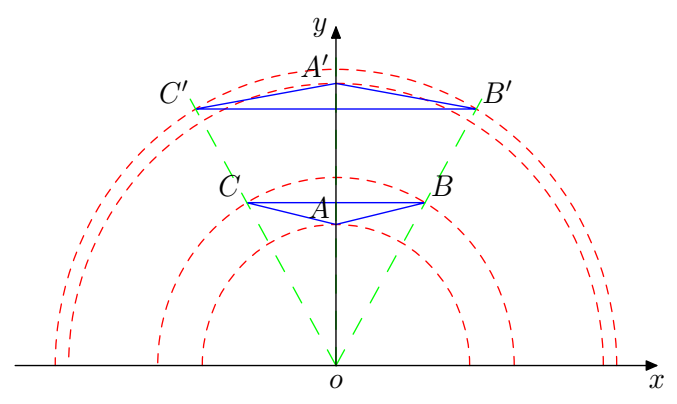

(a) Failure of affine element.

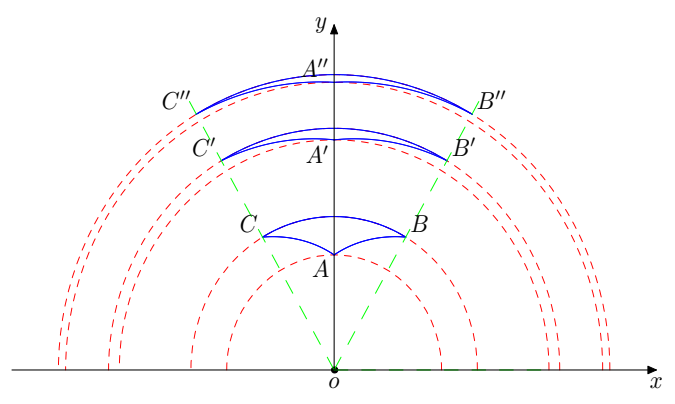

(b) Quadratic iso-parametric element.

Figure 1: Quadratic FE is superior in orientation-preservation.

To have an intuitive view of orientation-preserving behavior of finite element approximation of cavitation solutions, we compare schematically in Figure 1 the affine finite element interpolations and the quadratic iso-parametric finite element interpolations of a section of a ring before and after a large radially expansionary deformation. It is clearly seen in Figure 1(a) that the affine finite element interpolation fails to preserve the orientation, i.e., the interpolation triangle $A B C$ before the deformation is anticlockwise but the interpolation triangle $A^{\prime} B^{\prime} C^{\prime}$ after the deformation is clockwise. It is also easily seen in Figure 1(b) that the quadratic iso-parametric finite element interpolations can successfully preserve the 
orientation even for much larger deformations. This suggests that the conforming affine finite element method is not a good candidate for the cavitation computation, while quadratic finite element methods might be. In fact, for the conforming affine finite element method to preserve the orientation, the amount of degrees of freedom required can be unbearably large [25]. On the other hand, some numerical methods based on quadratic finite elements $[10,11]$, non-conforming affine finite element [25], bi-linear and tri-linear finite elements (see [13, 23] among many others) have shown considerable numerical success. In particular, the iso-parametric finite element method developed in [11] showed great potential in the computation of multi-voids growth, and the numerical experiments also revealed that the orientation-preservation conditions are crucial for the method to produce efficiently accurate finite element cavitation solutions.

The only practical analytical result on the orientation preservation condition for the cavitation computation known to the authors so far is [22], where a sufficient condition was given for a dual-parametric bi-quadratic finite element method.

In this paper, we study the orientation-preserving behavior of the quadratic iso-parametric finite element approximations of cavity solutions by analyzing the sufficient and necessary conditions for the interpolation functions to preserve the orientation. We will see that, compared with the dual-parametric bi-quadratic FEM, the derivation of the orientation preservation conditions for the quadratic iso-parametric FEM is more involved. Since the cavitation solutions are generally considered to vary mildly except in a neighborhood of the voids, where the material experiences large expansion dominant deformations, and where the difficulty of the computation as well as the analysis lies, we restrict ourselves to a simplified problem with $\Omega_{\varrho}=B_{1}(0) \backslash B_{\varrho}(0)$ in $\mathbb{R}^{2}$. To bring out the principal relations in the orientation-preservation conditions and avoid unnecessarily tedious calculations, we further restrict ourselves to simple expansionary boundary conditions of the form $u_{0}=\lambda x$ and the radially symmetric cavitation solutions. The result shows that the orientation-preservation can be achieved with a reasonable number of total degrees of freedom. In fact, combined with the corresponding interpolation error estimates, it would lead to an optimal meshing strategy, which we will show in a 
separate article [21].

The structure of the paper is as follows. In $\S 2$, we present some properties of the cavitation solutions for a specific class of energy functionals, as well as the quadratic iso-parametric finite element and a radially symmetric large expansion accommodating triangulation method. $\S 3$ is devoted to deriving the sufficient and necessary orientation-preservation conditions on the mesh distribution. We end the paper with some discussions and conclusion remarks in $\S 4$.

\section{Preliminaries}

We consider a typical class of stored energy density functions of the form

$$
W(F)=\Phi\left(v_{1}, \ldots, v_{n}\right)=\omega\left(\sum_{i=1}^{n} v_{i}^{2}\right)^{\frac{p}{2}}+g\left(\prod_{i=1}^{n} v_{i}\right), \quad \forall F \in M_{+}^{n \times n},
$$

where $\omega>0$ is a material constant, $v_{1}, \ldots, v_{n}$ are the singular values of the deformation gradient $F$, and where, to ensure the existence and regularity of cavity solutions [15], $n-1<p<n$, and $g:(0, \infty) \rightarrow[0, \infty)$ is a continuously differentiable strictly convex function satisfying

$$
g(d) \rightarrow+\infty \text { as } d \rightarrow 0 \text {, and } \frac{g(d)}{d} \rightarrow+\infty \text { as } d \rightarrow+\infty .
$$

For example, $g(d)=\frac{\chi}{2}(d-1)^{2}+\frac{1}{d}$ was used in [12] with the constant $\chi>0$ as the bulk modulus. As mentioned in the introduction, for simplicity, we henceforth assume that $n=2$.

\subsection{Properties of radially symmetric cavitation solutions}

For the simple expansionary boundary condition given by $u_{0}=\lambda x, \lambda>1$, and the

radially symmetric deformations $u(x)=\frac{r(|x|)}{|x|} x$, the problem defined on the domain $\Omega_{\varrho}=B_{1}(0) \backslash B_{\varrho}(0)$ reduces to minimizing the energy of the form

$$
I_{\varrho}(r)=\int_{\varrho}^{1} R \Phi\left(r^{\prime}(R), \frac{r(R)}{R}\right) d R
$$


in the set of admissible functions

$$
A_{\varrho}^{\lambda}=\left\{r \in W^{1,1}(\varrho, 1): r(\varrho)>0, r(1)=\lambda, \text { and } r^{\prime}>0 \text { a.e. }\right\} .
$$

It is well known (see, e.g. [17]) that the problem admits a unique minimizer $r_{\varrho}^{\lambda} \in C^{2}((\varrho, 1])$, which satisfies the Euler-Lagrange equation:

$$
\begin{gathered}
\frac{d}{d R}\left(R \Phi_{, 1}\left(r^{\prime}(R), \frac{r(R)}{R}\right)\right)=\Phi_{, 2}\left(r^{\prime}(R), \frac{r(R)}{R}\right), \quad R \in(\varrho, 1), \\
\omega p\left(r^{\prime}(\varrho)^{2}+\frac{r(\varrho)^{2}}{\varrho^{2}}\right)^{\frac{p}{2}-1} \frac{r^{\prime}(\varrho) \varrho}{r(\varrho)}+g^{\prime}(d(\varrho))=0, \\
r(1)=\lambda,
\end{gathered}
$$

where $d(\varrho)=\left.\operatorname{det} \nabla u\right|_{\partial B_{\varrho}(0)}=\frac{r(\varrho)}{\varrho} r^{\prime}(\varrho)$. In particular, for the perfect model $(\varrho=0)$, there exists a constant $\lambda_{c}>1$, such that, for $\lambda>\lambda_{c}$, the minimizer satisfies $r_{0}^{\lambda}(0)>0$; for $\lambda \leq \lambda_{c}$, the minimizer is given by $r_{0}^{\lambda}(R)=\lambda R$. Thus, by [17], for $\varrho>0, r_{\varrho}^{\lambda}(R) \geq r_{0}^{\lambda}(0)$ when $\lambda>\lambda_{c}$.

In the case of the perfect model, the radially symmetric cavity solution $r_{0}(R)$ is proved to be a bounded strictly convex function (see [17]), furthermore, it can be shown that $r_{0}(R)$ satisfies $m_{0} R \leq r_{0}^{\prime}(R) \leq M_{0} R$, for all $R \in(0,1]$, where $0<m_{0}<M_{0}$ are constants. The result is in fact valid also for the deficiency model, at least when $\varrho$ is sufficiently small.

Lemma 2.1 Let $r(R)$ be the minimizer of (2.3) over $A_{\varrho}^{\lambda}$ with the energy density function given by (2.1). If $\lambda>\lambda_{c}$, then for sufficiently small $\varrho \geq 0, r(R)$ satisfies

$$
0<r^{\prime \prime}(R) \leq C, \quad m R \leq r^{\prime}(R) \leq M R, \quad \forall R \in[\varrho, 1]
$$

where $C>0,0<m<M$ are constants independent of $\varrho$.

Proof. Firstly, a direct manipulation on (2.4) yields

$$
r^{\prime \prime}(R)=\frac{1}{R \Phi_{, 11}}\left(\frac{r(R)}{R}-r^{\prime}(R)\right)\left(\frac{\Phi_{, 2}-\Phi_{, 1}}{\frac{r(R)}{R}-r^{\prime}(R)}+\Phi_{, 12}\right) .
$$

It is straightforward to show that $\Phi_{, 11}$ and the term in the second bracket are always positive. With the same arguments as in [17], the term $\frac{r(R)}{R}-r^{\prime}(R)$ is 
either identically 0 or never vanishes. If $\frac{r(R)}{R}-r^{\prime}(R) \equiv 0$, then $r(R)=\lambda R$, which contradicts the fact that $r_{\varrho}^{\lambda}(R) \rightarrow r_{0}^{\lambda}(R)>0$ when $\lambda>\lambda_{c}$. On the other hand, it follows from $(2.5)$ that $g^{\prime}(d(\varrho)) \leq 0$. Since $g^{\prime \prime}(d)>0$, this yields $d(\varrho)=\frac{r^{\prime}(\varrho) r(\varrho)}{\varrho} \leq$ $d_{0}$, or equivalently $r^{\prime}(\varrho) \leq \frac{d_{0} \varrho}{r(\varrho)}$, where $d_{0}$ is the unique root of $g^{\prime}(x)=0$ (see $\left.(2.2)\right)$. Thus, for the cavity solution, we have $\frac{r(\varrho)}{\varrho}-r^{\prime}(\varrho) \geq \frac{r_{c}}{\varrho}-\frac{d_{0} \varrho}{r_{c}}>0$, where $r_{c}=r_{0}^{\lambda}(0)$. Hence $\frac{r(R)}{R}-r^{\prime}(R)>0$ and consequently $r^{\prime \prime}(R)>0$, as long as $\varrho<\frac{r_{c}}{\sqrt{d_{0}}}$.

Next, we notice that the radial component of the Cauchy stress $T(R)=\frac{R}{r(R)} \Phi_{, 1}($ $\left.r^{\prime}(R), \frac{r(R)}{R}\right)$ is nowhere decreasing $([17])$, and $T(\varrho)=0$. Hence $T(R) \geq 0$ for $R \in[\varrho, 1]$, which can be reformulated as

$$
g^{\prime}(d(R)) \geq-\omega p\left(r^{\prime}(R)^{2}+\frac{r(R)^{2}}{R^{2}}\right)^{\frac{p}{2}-1} \frac{r^{\prime}(R) R}{r(R)}, \quad \forall R \in[\varrho, 1) .
$$

Since $p \in(1,2), r^{\prime}(R) R<r(R)$, and $r(R) \geq r_{c}$, this yields $g^{\prime}(d(R)) \geq-\omega p r_{c}^{p-2}$. Thus, by the convexity of $g$ and $r(R) \leq r(1)=\lambda$, we obtain $r^{\prime}(R) \geq \frac{d_{-}}{\lambda} R$, where $d_{-} \in\left(0, d_{0}\right)$ is such that $g^{\prime}\left(d_{-}\right)=-\omega p r_{c}^{p-2}$. On the other hand, we notice that $r(R) \geq r_{0}(R)$ (see [19]), and consequently $T_{0}(R) \geq T(R)$, for all $R \in[\varrho, 1)$ and $\varrho>0$, where $r_{0}(R)$ is the cavity solution of the perfect model,

$$
T_{0}(R)=\omega p\left(r_{0}^{\prime}(R)^{2}+\frac{r_{0}(R)^{2}}{R^{2}}\right)^{\frac{p}{2}-1} \frac{r_{0}^{\prime}(R) R}{r_{0}(R)}+g^{\prime}\left(d_{0}(R)\right)
$$

is the normal surface traction with respect to the perfect model, and $d_{0}(R)=$ $\frac{r_{0}^{\prime}(R) r_{0}(R)}{R}$. Thus, we have

$$
g^{\prime}(d(R)) \leq g^{\prime}\left(d_{0}(R)\right)+\omega p\left(r_{0}^{\prime}(R)^{2}+\frac{r_{0}(R)^{2}}{R^{2}}\right)^{\frac{p}{2}-1} \frac{r_{0}^{\prime}(R) R}{r_{0}(R)} \leq g^{\prime}\left(d_{0}(R)\right)+\frac{\omega p}{r_{c}^{2-p}}
$$

since $r(R) \geq r_{0}(R) \geq r_{c}$ (see [17]) and $r_{0}^{\prime}(R)<\frac{r_{0}(R)}{R}$. Denote $d_{0+}=\max _{0 \leq R \leq 1} d_{0}(R)$ and $d_{+}=\left(g^{\prime}\right)^{-1}\left(g^{\prime}\left(d_{0+}\right)+\omega p r_{c}^{p-2}\right)$. Then the above inequality yields $\bar{d}(R) \leq d_{+}$, and consequently $r^{\prime}(R) \leq \frac{d_{+}}{r_{c}} R$. Hence, $m=\frac{d_{-}}{\lambda}$ and $M=\frac{d_{+}}{r_{c}}$ in (2.7).

The uniform boundedness of $r^{\prime \prime}(R)$ can be verified directly by (2.8) using the facts that $0<d_{-} \leq d(R) \leq d_{+}, 0<r_{c} \leq r(R) \leq \lambda$ and $m R \leq r^{\prime}(R) \leq M R$. 


\subsection{The quadratic iso-parametric FEM}

Let $(\hat{T}, \hat{P}, \hat{\Sigma})$ be a quadratic Lagrange reference element. Define $F_{T}: \hat{T} \rightarrow \mathbb{R}^{2}$

$$
\left\{\begin{array}{l}
F_{T} \in\left(P_{2}(\hat{T})\right)^{2} \\
x=F_{T}(\hat{x})=\sum_{i=1}^{3} a_{i} \hat{\mu}_{i}(\hat{x})+\sum_{1 \leq i<j \leq 3} a_{i j} \hat{\mu}_{i j}(\hat{x}),
\end{array}\right.
$$

where $a_{i}, 1 \leq i \leq 3$, and $a_{i j}, 1 \leq i<j \leq 3$ are given points in $\mathbb{R}^{2}$, and

$$
\hat{\mu}_{i}(\hat{x})=\hat{\lambda}_{i}(\hat{x})\left(2 \hat{\lambda}_{i}(\hat{x})-1\right), \quad \hat{\mu}_{i j}(\hat{x})=4 \hat{\lambda}_{i}(\hat{x}) \hat{\lambda}_{j}(\hat{x}),
$$

with $\hat{\lambda}_{i}(\hat{x}), 1 \leq i \leq 3$ being the barycentric coordinates of $\hat{T}$. If the map $F_{T}$ defined above is an injection, then $T=F_{T}(\hat{T})$ is a curved triangular element as shown in Figure 3. The standard quadratic iso-parametric finite element is defined as a finite element triple $\left(T, P_{T}, \Sigma_{T}\right)$ with

$$
\left\{\begin{array}{l}
T=F_{T}(\hat{T}) \text { being a curved triangle element, } \\
P_{T}=\left\{p: T \rightarrow \mathbb{R}^{2} \mid p=\hat{p} \circ F_{T}^{-1}, \hat{p} \in \hat{P}\right\}, \\
\Sigma_{T}=\left\{p\left(a_{i}\right), 1 \leq i \leq 3 ; p\left(a_{i j}\right), 1 \leq i<j \leq 3\right\} .
\end{array}\right.
$$

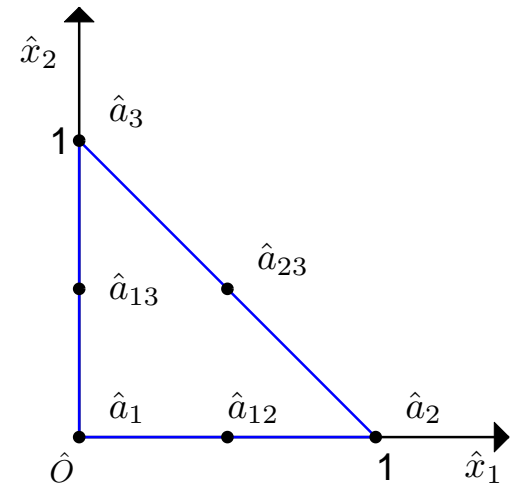

Figure 2: The reference element $\hat{T}$. Figure 3: A curved triangular element $\mathrm{T}$.

\subsection{Large expansion accommodating triangulations}

Let $\hat{\mathcal{J}}$ be a straight edged triangulation on $\Omega_{\varrho}=B_{1}(0) \backslash B_{\varrho}(0)$, and let $1>$ $\mu>\varrho$ be given constants. For a triangular element $\hat{K} \in \hat{\mathcal{J}}$ with vertices $a_{i}, 1 \leq$ 
$i \leq 3$, to accommodate the large expansionary deformation around the defect and approximate the curved boundary better, choose $a_{i j}$ in the following way: if $a_{i}$, $a_{j} \in\{x: \mu<|x|<1\}$, then $a_{i j}$ is set as the midpoint of $a_{i}$ and $a_{j}$; otherwise denote $(r(x), \theta(x))$ the polar coordinates of $x$, then set

$$
a_{i j}=\left(r_{i j} \cos \theta_{i j}, r_{i j} \sin \theta_{i j}\right),
$$

where

$$
r_{i j}=\frac{r\left(a_{i}\right)+r\left(a_{j}\right)}{2}, \quad \theta_{i j}=\frac{\theta\left(a_{i}\right)+\theta\left(a_{j}\right)}{2} .
$$

With the six points $a_{i}$ and $a_{i j}$, a triangular element $K$ is defined by the mapping (2.9). With this kind of curved elements in a neighborhood of the defects and on the outer boundary, while using general straight triangles elsewhere, the mesh can better accommodate the locally large expansionary deformations.

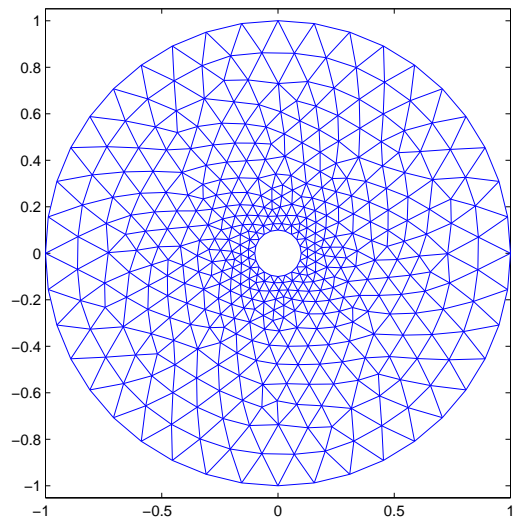

Figure 4: An EasyMesh $\hat{\mathcal{J}}^{\prime}$.

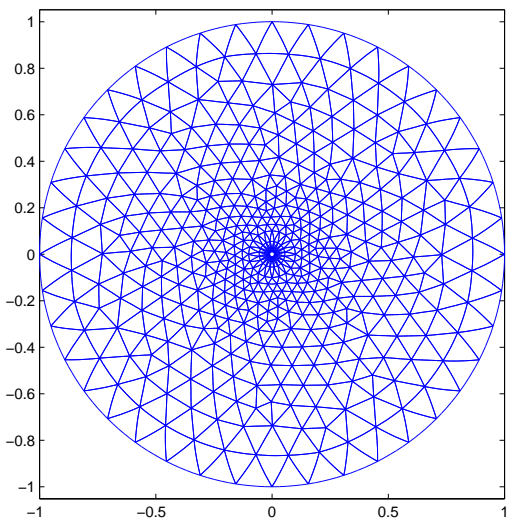

Figure 5: $\mathcal{J}$, a mesh adapted to cavity.

When the radius of the defect $\varrho$ is very small, the mesh produced by the EasyMesh (a software producing 2-d triangular mesh [11]) can be irregular near the defect. To produce a mesh which can better accommodate a void growth, as suggested by [11], a mesh $\hat{\mathcal{J}}^{\prime}$ can be introduced on $\Omega_{\hat{\varrho}}$ with $\varrho$ $\gg \varrho$ by the EasyMesh, which is then transformed as $\mathcal{J}^{\prime}$ under the iso-parametric deformation as above and coupled with several layers of circumferentially uniform mesh $\mathcal{J}^{\prime \prime}$ given on the domain $\{x: \varrho \leq|x| \leq \hat{\varrho}\}$, where on each layer the mesh is similar to that shown in Figure 6 (also see therein for a more specific description), to produce a final mesh 
$\mathcal{J}=\mathcal{J}^{\prime} \cup \mathcal{J}^{\prime \prime}$. As an example, an EasyMesh produced mesh $\hat{\mathcal{J}}^{\prime}$ with $\hat{\varrho}=0.1$ is shown in Figure 4, and the final mesh $\mathcal{J}$ with $\varrho=0.01, \mu=0.15$, and a two-layer circumferentially uniform mesh $\mathcal{J}^{\prime \prime}$ is shown in Figure 5.

\section{On the orientation-preservation conditions}

We are concerned with orientation-preservation of large expansionary finite element deformations around a small prescribed void. A typical curved triangulation around a prescribed circular ring with inner radius $\epsilon=0.01$ and thickness $\tau=0.01$ is shown in Figure 6, in which we see that the curved triangular elements can be classified into two basic types, namely types $\mathrm{A}$ and B. More precisely, let $N$ be the number of evenly spaced nodes on both circles, then, each of the types A and B elements $A_{i}, B_{i}, i=0,1,2, \cdots, N-1$, are defined by three nodes, denoted as $a_{i, j}^{A}=\left(r_{i, j}^{A}, \theta_{i, j}^{A}\right)$ and $a_{i, j}^{B}=\left(r_{i, j}^{B}, \theta_{i, j}^{B}\right), j=1,2,3$, in polar coordinates, as follows:

$$
\begin{aligned}
r_{i, 1}^{A}=r_{i, 2}^{B}=r_{i, 3}^{B}=\epsilon, & r_{i, 2}^{A}=r_{i, 3}^{A}=r_{i, 1}^{B}=\epsilon+\tau \\
\theta_{i, 1}^{A}=\theta_{i, 3}^{B}=\theta_{i-1,2}^{B}=\frac{2 i}{N} \pi, & \theta_{i, 2}^{A}=\theta_{i-1,3}^{A}=\theta_{i-1,1}^{B}=\frac{2 i-1}{N} \pi
\end{aligned}
$$

where the first subscript in $r$ and $\theta$ is understood in the sense of $\bmod (N)$.

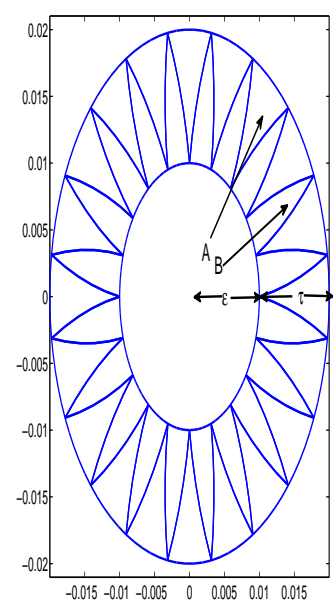

Figure 6: A typical layer of circumferentially uniform curved triangulation. 
To have a better picture in our mind for the problem given below, we first introduce some notations. Let $\epsilon$ and $\tau$ represent respectively the inner radius and the thickness of the circular annulus as shown in Figure 6 . Let $2 N$ be the number of the elements in the circular annulus, and denote $\kappa \triangleq \epsilon / \tau, \Omega_{(\epsilon, \tau)}=\left\{x \in \mathbb{R}^{2}\right.$ : $\epsilon \leq|x| \leq \epsilon+\tau\}$. Throughout the paper, we use the notation $\Phi \preceq \Psi$ to mean that there exists a generic constant $C$ independent of $\epsilon, \tau$ such that $|\Phi| \leq C \Psi$. And $\Phi \sim \Psi$ means that $\Psi \preceq \Phi \preceq \Psi$.

Let $u$ be the cavitation solution, and let $\mathcal{J}=\mathcal{J}^{\prime} \cup \mathcal{J}^{\prime \prime}$ be a given mesh (see $\S 2$ ) with the layers in $\mathcal{J}^{\prime \prime}$ consisting of well defined curved triangular elements of types A and B satisfying det $\nabla x>0$ (see Corollary 3.5 for details). To have $u$ well resolved by functions in the finite element function space defined on $\mathcal{J}$, a necessary condition is that the finite element interpolation function $\Pi u(x)$ is an admissible function. Since $u$ is considered regular elsewhere other than in $\mathcal{J}^{\prime \prime}$, where the material is subjected to a locally large expansion dominant deformation, and since the key for a finite element interpolation function to be admissible is $\operatorname{det} \nabla \Pi u(x)>0$ on each of the curved triangular element, for simplicity and without loss of generality, we will investigate in this section the conditions that ensure $\operatorname{det} \nabla \Pi u(x)>0$ for radially symmetric expansionary deformations of the form $u(x)=\frac{r(|x|)}{|x|} x$. Since $\operatorname{det} \nabla \Pi u(x) \cdot \operatorname{det} \nabla x=\operatorname{det} \frac{\partial \Pi u}{\partial \hat{x}}$, it suffices to ensure $\operatorname{det} \frac{\partial \Pi u}{\partial \hat{x}}>0$ and $\operatorname{det} \nabla x>0$ on the curved triangular elements of types A and B in all layers (see $\S 2.3$ ).

The two lemmas below are the main ingredients for the orientation-preservation conditions. To simplify the notations, for any positive function $s(\cdot)$, we denote $s_{0}=s(\epsilon), s_{1 / 2}=s(\epsilon+\tau / 2), s_{1}=s(\epsilon+\tau), \kappa_{0}^{s}=\frac{s(\epsilon)}{s(\epsilon+\tau)}$, and $\kappa_{1 / 2}^{s}=\frac{s\left(\epsilon+\frac{\tau}{2}\right)}{s(\epsilon+\tau)}$.

We first give the orientation-preservation conditions for the type A elements.

Lemma 3.1 Let $v(x)=\frac{s(|x|)}{|x|} x$, where $s(t)$ is a positive function satisfying $s^{\prime}(t)>$ $0, s^{\prime \prime}(t) \geq 0, \forall t \in(0,1]$, and

$$
2 s_{1 / 2}>s_{1} .
$$

Then, the Jacobian determinant $\operatorname{det} \frac{\partial \Pi v\left(F_{T}(\hat{x})\right)}{\partial \hat{x}}$ of the iso-parametric finite element interpolation function $\Pi v\left(F_{T}(\hat{x})\right)$ is positive on the elements of type $A$ in $\Omega_{(\epsilon, \tau)}$ if 
and only if

$$
-3 s_{0}-s_{1} \cos \frac{\pi}{N}+4 s_{1 / 2} \cos \frac{\pi}{2 N}>0,
$$

and

$$
-6 s_{1} \cos ^{3} \frac{\pi}{2 N}+4 s_{1 / 2} \cos ^{2} \frac{\pi}{2 N}+\left(s_{0}+9 s_{1}\right) \cos \frac{\pi}{2 N}-8 s_{1 / 2}>0 .
$$

Proof. For the radially symmetric function $v(x)=\frac{s(|x|)}{|x|} x$, the iso-parametric finite element interpolation function can be written as (see $\S 2$ )

$$
\Pi v(x)=\sum_{i=1}^{3} b_{i} \hat{\mu}_{i}(\hat{x})+\sum_{1 \leq i<j \leq 3} b_{i j} \hat{\mu}_{i j}(\hat{x})
$$

where $\hat{x}=F_{T}^{-1}(x)$, and where, for a representative of type A element, $b_{1}=\left(s_{0}, 0\right)$, $b_{2}=\left(s_{1} \cos \frac{\pi}{N},-s_{1} \sin \frac{\pi}{N}\right), b_{3}=\left(s_{1} \cos \frac{\pi}{N}, s_{1} \sin \frac{\pi}{N}\right), b_{12}=\left(s_{1 / 2} \cos \frac{\pi}{2 N},-s_{1 / 2} \sin \frac{\pi}{2 N}\right)$, $b_{13}=\left(s_{1 / 2} \cos \frac{\pi}{2 N}, s_{1 / 2} \sin \frac{\pi}{2 N}\right), b_{23}=\left(s_{1}, 0\right)$. On this element, we have

$$
\Pi v(x)=\left(s_{0}+\alpha_{2} y+2 \alpha_{1} y^{2}-4 s_{1} \sin ^{2} \frac{\pi}{2 N}\left(\hat{x}_{1}^{2}+\hat{x}_{2}^{2}\right),(2 \gamma y-\beta)\left(\hat{x}_{2}-\hat{x}_{1}\right)\right),
$$

where $y=\hat{x}_{1}+\hat{x}_{2}$,

$$
\begin{aligned}
\alpha_{1} & =s_{0}+s_{1}-2 s_{1 / 2} \cos \frac{\pi}{2 N}, \\
\alpha_{2} & =-3 s_{0}-s_{1} \cos \frac{\pi}{N}+4 s_{1 / 2} \cos \frac{\pi}{2 N}, \\
\beta & =s_{1} \sin \frac{\pi}{N}-4 s_{1 / 2} \sin \frac{\pi}{2 N}, \\
\gamma & =s_{1} \sin \frac{\pi}{N}-2 s_{1 / 2} \sin \frac{\pi}{2 N} .
\end{aligned}
$$

Hence

$$
\frac{\partial \Pi v}{\partial \hat{x}}=\left(\begin{array}{cc}
\alpha-8 s_{1} \hat{x}_{1} \sin ^{2} \frac{\pi}{2 N} & \alpha-8 s_{1} \hat{x}_{2} \sin ^{2} \frac{\pi}{2 N} \\
\beta-4 \gamma \hat{x}_{1} & -\beta+4 \gamma \hat{x}_{2}
\end{array}\right),
$$

where $\alpha=4 \alpha_{1} y+\alpha_{2}$. It follows that

$$
\begin{aligned}
\operatorname{det} \frac{\partial \Pi v}{\partial \hat{x}}\left(\hat{x}_{1}, \hat{x}_{2}\right)=H(y, z) \triangleq 16 \gamma \alpha_{1} y^{2}-64 s_{1} \gamma \sin ^{2} \frac{\pi}{2 N} z \\
+\left(-8 \beta\left(\alpha_{1}-s_{1} \sin ^{2} \frac{\pi}{2 N}\right)+4 \gamma \alpha_{2}\right) y-2 \beta \alpha_{2},
\end{aligned}
$$


where $z=\hat{x}_{1} \hat{x}_{2}$. Note that $\operatorname{det} \frac{\partial \Pi v}{\partial \hat{x}}>0$ on $\hat{T}=\hat{T}_{1} \cup \hat{T}_{2}$, where $\hat{T}_{1}=\left\{\left(\hat{x}_{1}, \hat{x}_{2}\right): 0 \leq\right.$ $\left.\hat{x}_{2} \leq 1 / 2, \hat{x}_{2} \leq \hat{x}_{1} \leq 1-\hat{x}_{2}\right\}$ and $\hat{T}_{2}=\left\{\left(\hat{x}_{1}, \hat{x}_{2}\right): 0 \leq \hat{x}_{1} \leq 1 / 2, \hat{x}_{1} \leq \hat{x}_{2} \leq 1-\hat{x}_{1}\right\}$, is equivalent to $H(y, z)>0$ on the domain $\left\{0 \leq y \leq 1,0 \leq z \leq \frac{y^{2}}{4}\right\}$.

Firstly, (3.2) follows from $H(0,0)=-2 \beta \alpha_{2}>0$ and $\beta<0$, and (3.3) is a direct result of $H(1,0)=4 s_{1} \sin \frac{\pi}{2 N}\left(-6 s_{1} \cos ^{3} \frac{\pi}{2 N}+4 s_{1 / 2} \cos ^{2} \frac{\pi}{2 N}+\left(s_{0}+9 s_{1}\right) \cos \frac{\pi}{2 N}-\right.$ $\left.8 s_{1 / 2}\right)>0$.

Conversely, we infer from (3.2) and (3.3) that $H(y, z)>0$ for $0 \leq y \leq 1$, $0 \leq z \leq \frac{y^{2}}{4}$ as following.

(i) If $\cos \frac{\pi}{2 N}>\kappa_{1 / 2}^{s}$, i.e., $\gamma>0$, then $\frac{\partial H}{\partial z}<0$. Thus, it suffices to show $H(y, z)>0$ on the curve $z=y^{2} / 4(0 \leq y \leq 1)$. On this curve, we have

$$
\begin{aligned}
H(y, z) & =G(y) \triangleq 16 \gamma \alpha_{3} y^{2}+\left(-8 \beta \alpha_{3}+4 \gamma \alpha_{2}\right) y-2 \beta \alpha_{2} \\
& =2(2 \gamma y-\beta)\left(4 \alpha_{3} y+\alpha_{2}\right),
\end{aligned}
$$

where

$$
\alpha_{3}=s_{1} \cos ^{2} \frac{\pi}{2 N}-2 s_{1 / 2} \cos \frac{\pi}{2 N}+s_{0} .
$$

Consider the sign of $\alpha_{3}$. If $\alpha_{3}>0$, then by (3.2), both roots $y_{1}=\frac{\beta}{2 \gamma}$ and $y_{2}=-\frac{\alpha_{2}}{4 \alpha_{3}}$ of the equation $G(y)=0$ are negative. So, it follows from $G^{\prime \prime}(y)=32 \gamma \alpha_{3}>0$ that $G(y)>0$ for $y>0$.

While if $\alpha_{3} \leq 0$, we have $G(0)>0, G^{\prime \prime}(y) \leq 0$ and, recalling that $s>0$, $s^{\prime}>0$ and $s^{\prime \prime} \geq 0$,

$$
\begin{aligned}
G(1) & =2(2 \gamma-\beta)\left(2 s_{1} \cos ^{2} \frac{\pi}{2 N}-4 s_{1 / 2} \cos \frac{\pi}{2 N}+s_{0}+s_{1}\right), \\
& \geq 2(2 \gamma-\beta)\left(2 s_{1 / 2} \cos ^{2} \frac{\pi}{2 N}-4 s_{1 / 2} \cos \frac{\pi}{2 N}+2 s_{1 / 2}\right) \\
& =4(2 \gamma-\beta) s_{1 / 2}\left(\cos \frac{\pi}{2 N}-1\right)^{2}>0,
\end{aligned}
$$

as a consequence, we infer that $G(y)>0$ on $[0,1]$.

(ii) If $\cos \frac{\pi}{2 N}=\kappa_{1 / 2}^{s}$, i.e., $\gamma=0$, then, $H(y, z)=-2 \beta\left(4 \alpha_{3} y+\alpha_{2}\right)$ with $\beta<$ 0 . A similar argument as in (i) yields $H(0, z)=-2 \beta \alpha_{2}>0, H(1, z)=$ $-2 \beta\left(\alpha_{2}+4 \alpha_{3}\right)>0$, hence we conclude $H(y, z)$ is positive on the domain $\left\{0 \leq y \leq 1,0 \leq z \leq \frac{y^{2}}{4}\right\}$. 
(iii) If $\cos \frac{\pi}{2 N}<\kappa_{1 / 2}^{s}$, i.e., $\gamma<0$, and thus $\frac{\partial H(y, z)}{\partial z}>0$, it is sufficient to guarantee $H(y, z)>0$ on the set $\{z=0,0 \leq y \leq 1\}$. On the curve $z=0$,

$$
H(y, 0)=G(y) \triangleq 16 \gamma \alpha_{1} y^{2}+\left(-8 \beta \alpha_{3}+4 \gamma \alpha_{2}\right) y-2 \beta \alpha_{2} .
$$

Since $\gamma<0$, and $s>0, s^{\prime \prime} \geq 0$ implies $\alpha_{1}>0$, it follows that $G^{\prime \prime}(y)<0$. Thus, $G(y)>0$ for $y \in[0,1]$ equivalents to $G(0)>0$ and $G(1)>0$.

By (3.2) and $\beta<0, G(0)>0$. On the other hand, it follows from (3.3) that $G(1)=4 s_{1} \sin \frac{\pi}{2 N}\left(-6 s_{1} \cos ^{3} \frac{\pi}{2 N}+4 s_{1 / 2} \cos ^{2} \frac{\pi}{2 N}+\left(s_{0}+9 s_{1}\right) \cos \frac{\pi}{2 N}-8 s_{1 / 2}\right)>0$.

Thus we are led to the conclusion.

Similarly, the sufficient and necessary condition for the elements of type B is as follows.

Lemma 3.2 Under the same assumptions of Lemma 3.1, the Jacobian determinant det $\frac{\partial \Pi v\left(F_{T}(\hat{x})\right)}{\partial \hat{x}}$ of the iso-parametric finite element interpolation function $\Pi v\left(F_{T}(\hat{x})\right)$ is positive on the curved elements of type $B$ in the circular domain $\Omega_{(\epsilon, \tau)}$ if and only if

$$
2 s_{0} \cos ^{2} \frac{\pi}{2 N}-4 s_{1 / 2} \cos \frac{\pi}{2 N}+s_{0}+s_{1}<0 .
$$

Proof. Consider $\Pi v(x)$ defined by (3.4) on a representative element of type B with $b_{1}=\left(s_{1}, 0\right), b_{2}=\left(s_{0} \cos \frac{\pi}{N}, s_{0} \sin \frac{\pi}{N}\right), b_{3}=\left(s_{0} \cos \frac{\pi}{N},-s_{0} \sin \frac{\pi}{N}\right), b_{12}=$ $\left(s_{1 / 2} \cos \frac{\pi}{2 N}, s_{1 / 2} \sin \frac{\pi}{2 N}\right), b_{13}=\left(s_{1 / 2} \cos \frac{\pi}{2 N},-s_{1 / 2} \sin \frac{\pi}{2 N}\right), b_{23}=\left(s_{0}, 0\right)$. On this element, one has

$$
\Pi v(x)=\left(s_{1}+\bar{\alpha}_{2} y+2 \bar{\alpha}_{1} y^{2}-4 s_{0} \sin ^{2} \frac{\pi}{2 N}\left(\hat{x}_{1}^{2}+\hat{x}_{2}^{2}\right),(2 \bar{\gamma} y-\bar{\beta})\left(\hat{x}_{1}-\hat{x}_{2}\right)\right),
$$

where

$$
\begin{aligned}
\bar{\alpha}_{1} & =s_{0}+s_{1}-2 s_{1 / 2} \cos \frac{\pi}{2 N}, \\
\bar{\alpha}_{2} & =-3 s_{1}-s_{0} \cos \frac{\pi}{N}+4 s_{1 / 2} \cos \frac{\pi}{2 N}, \\
\bar{\beta} & =s_{0} \sin \frac{\pi}{N}-4 s_{1 / 2} \sin \frac{\pi}{2 N}, \\
\bar{\gamma} & =s_{0} \sin \frac{\pi}{N}-2 s_{1 / 2} \sin \frac{\pi}{2 N} .
\end{aligned}
$$


Hence

$$
\begin{gathered}
\frac{\partial \Pi v}{\partial \hat{x}}=\left(\begin{array}{cc}
\bar{\alpha}-8 s_{0} \hat{x}_{1} \sin ^{2} \frac{\pi}{2 N} & \bar{\alpha}-8 s_{0} \hat{x}_{2} \sin ^{2} \frac{\pi}{2 N} \\
-\bar{\beta}+4 \bar{\gamma} \hat{x}_{1} & \bar{\beta}-4 \bar{\gamma} \hat{x}_{2}
\end{array}\right), \\
\operatorname{det} \frac{\partial \Pi v}{\partial \hat{x}}=H(y, z)=-16 \bar{\gamma} \bar{\alpha}_{1} y^{2}+64 s_{0} \bar{\gamma} \sin ^{2} \frac{\pi}{2 N} z+\left(8 \bar{\beta} \bar{\alpha}_{3}-4 \bar{\gamma} \bar{\alpha}_{2}\right) y+2 \bar{\beta} \bar{\alpha}_{2},
\end{gathered}
$$

where, recalling that $s>0, s^{\prime}>0$ and $s^{\prime \prime} \geq 0$, we get

$$
\begin{aligned}
\bar{\alpha}_{3} & =\bar{\alpha}_{1}-s_{0} \sin ^{2} \frac{\pi}{2 N} \\
& =s_{0} \cos ^{2} \frac{\pi}{2 N}-2 s_{1 / 2} \cos \frac{\pi}{2 N}+s_{1} \\
& \geq s_{0} \cos ^{2} \frac{\pi}{2 N}-\left(s_{0}+s_{1}\right) \cos \frac{\pi}{2 N}+s_{1} \\
& =\left(\cos \frac{\pi}{2 N}-1\right)\left(s_{0} \cos \frac{\pi}{2 N}-s_{1}\right)>0
\end{aligned}
$$

Since $\bar{\gamma}<0$, i.e., $\frac{\partial H(y, z)}{\partial z}<0$, thus, it suffices to guarantee $H(y, z)>0$ on the curve $z=y^{2} / 4$, for $0 \leq y \leq 1$. On this curve, we have

$$
\begin{aligned}
H\left(y, y^{2} / 4\right) & =G(y) \triangleq-16 \bar{\gamma} \bar{\alpha}_{3} y^{2}+\left(8 \bar{\beta} \bar{\alpha}_{3}-4 \bar{\alpha}_{2} \bar{\gamma}\right) y+2 \bar{\alpha}_{2} \bar{\beta} \\
& =-2(2 \bar{\gamma} y-\bar{\beta})\left(4 \bar{\alpha}_{3} y+\bar{\alpha}_{2}\right) .
\end{aligned}
$$

Let $y_{1}, y_{2}$ be the two roots of $G(y)=0$. Since $y_{1}=\frac{\bar{\beta}}{2 \bar{\gamma}}>1$ and $G^{\prime \prime}(y)=-32 \bar{\gamma} \bar{\alpha}_{3}>$ 0 , we see that $G(y)>0$ on $[0,1]$ equivalents to $y_{2}=\frac{-\bar{\alpha}_{2}}{4 \bar{\alpha}_{3}}>1$, or

$$
4 \bar{\alpha}_{3}+\bar{\alpha}_{2}=2 s_{0} \cos ^{2} \frac{\pi}{2 N}-4 s_{1 / 2} \cos \frac{\pi}{2 N}+s_{0}+s_{1}<0 .
$$

Hence, the proof is completed.

Remark 3.3 As is shown in the proof of Lemmas 3.1 and 3.2, $\operatorname{det} \frac{\partial \Pi v}{\partial \hat{x}}>0$ is satisfied on the elements if and only if $\operatorname{det} \frac{\partial \Pi v}{\partial \hat{x}}>0$ on the three vertices of the type A elements, while on the midpoint of the inner circle edge of the type B elements.

Theorem 3.4 Under the assumptions of Lemma 3.1, the Jacobian determinant $\operatorname{det} \frac{\partial \Pi v\left(F_{T}(\hat{x})\right)}{\partial \hat{x}}$ is positive on the curved elements in the circular domain $\Omega_{(\epsilon, \tau)}$ if and only if

$$
4 s_{1 / 2}>3 s_{0}+s_{1}
$$


and $\cos \frac{\pi}{2 N}>\max \left\{l_{1}, l_{2}\right\}$, where $l_{1}$ is the smaller root of the equation

$$
2 s_{0} z^{2}-4 s_{1 / 2} z+s_{0}+s_{1}=0
$$

$l_{2}$ is the second root of the equation

$$
-6 s_{1} z^{3}+4 s_{1 / 2} z^{2}+\left(s_{0}+9 s_{1}\right) z-8 s_{1 / 2}=0 .
$$

Proof. Firstly, the inequality (3.13) implies that $l_{1}<1$ as well as the bigger root of equation (3.14) is greater than 1. On the other hand, if (3.13) does not hold, then one has $l_{1} \geq 1$, consequently there is no $N$ such that (3.11) is satisfied. Next, let $l_{3}$ be the smaller root of the equation (see (3.2))

$$
2 s_{1} z^{2}-4 s_{1 / 2} z+3 s_{0}-s_{1}=0
$$

Then the inequalities (3.2), (3.3), (3.11) are satisfied if and only if $\cos \frac{\pi}{2 N}>$ $\max \left\{l_{1}, l_{2}, l_{3}\right\}$. Substituting $l_{3}$ into the left hand side of (3.14), we have

$$
2 s_{0} l_{3}^{2}-4 s_{1 / 2} l_{3}+s_{0}+s_{1}=2\left(s_{0}-s_{1}\right)\left(l_{3}^{2}-1\right)>0,
$$

which together with (3.13) implies $l_{3}<l_{1}$. Thus, the conclusion of the theorem follows from Lemmas 3.1 and 3.2.

The theorem allows us to work out an explicit condition for a mesh, defined on a ring region with curved triangular elements of types $\mathrm{A}$ and $\mathrm{B}$, to be well defined in the sense that $\operatorname{det} \frac{\partial x}{\partial \hat{x}}>0$.

Corollary 3.5 Let $\epsilon>0, \tau>0$, and $\kappa \triangleq \epsilon / \tau$. Let a layer of evenly spaced $N$ couples of curved triangular mesh elements of types $A$ and $B$ be introduced on the circular domain $\Omega_{(\epsilon, \tau)}$ by (2.9) and (2.11) (see Figure 6). Then, there exists an integer $\hat{N}(\kappa)$, such that, the Jacobian determinant of the mesh map is positive, i.e., $\operatorname{det} \frac{\partial x}{\partial \hat{x}}>0$, if and only if $N \geq \hat{N}(\kappa)$. Moreover, $\hat{N}=\hat{N}(\kappa) \sim 1+\kappa^{1 / 4}$.

Proof. Taking $v(x)=x$, or equivalently $s(t)=\mathrm{id}(t)=t$, in Lemma 3.1, then it is easily verified that (3.1) and (3.13) are satisfied. Consequently, by Theorem 3.4, we 
conclude that $\operatorname{det} \frac{\partial x}{\partial \hat{x}}>0$ on the elements if and only if $\cos \frac{\pi}{2 N}>\hat{l}(\kappa)=\max \left\{\hat{l}_{1}, \hat{l}_{2}\right\}$, where $\hat{l}_{1}$ is the smaller root of the equation

$$
\kappa z^{2}-(1+2 \kappa) z+\kappa+1 / 2=0
$$

$\hat{l}_{2}$ is the second root of the equation

$$
-6(1+\kappa) z^{3}+4(1 / 2+\kappa) z^{2}+(9+10 \kappa) z-8(1 / 2+\kappa)=0 .
$$

Thus the conclusion follows by setting $\hat{N} \triangleq\left[\frac{\pi}{2 \arccos \hat{l}(\kappa)}\right]+1$. What remains for us to show now is $\hat{N}=\hat{N}(\kappa) \sim 1+\kappa^{1 / 4}$.

If $\kappa=\epsilon / \tau$ is bounded above by a constant $C \geq 1$, note that $\hat{l}<\frac{\kappa+1 / 2}{\kappa+1} \leq \frac{1+2 C}{2+2 C}$, it follows that $\hat{N} \leq\left[\frac{\pi}{2 \arccos \frac{1+2 C}{2+2 C}}\right]+1$.

Next we consider the case when $\kappa>C$. Notice that $\cos \frac{\pi}{2 N}>\hat{l}(\kappa)$ is equivalent to $\sin ^{2} \frac{\pi}{4 N}<\frac{1-\hat{l}(\kappa)}{2}=\min \left\{\frac{1-\hat{l}_{1}(\kappa)}{2}, \frac{1-\hat{l}_{2}(\kappa)}{2}\right\}$, and for $\kappa \gg 1$, we have

$$
\frac{1-\hat{l}_{1}(\kappa)}{2}=\frac{1}{2(1+\sqrt{1+2 \kappa})}=\frac{\kappa^{-1 / 2}}{2 \sqrt{2}}+O\left(\kappa^{-1}\right) .
$$

On the other hand, since $\hat{l}_{2}(\kappa)$ is the second root of the equation $(3.17)$, then $\frac{1-\hat{l}_{2}(\kappa)}{2}$ is the second root of the equation

$$
-6(1+\kappa)(1-2 z)^{3}+4(\kappa+1 / 2)(1-2 z)^{2}+(9+10 \kappa)(1-2 z)-8(1 / 2+\kappa)=0 .
$$

Denote $t=\frac{1}{1+\kappa}$, then, the equation can be rewritten as

$$
z^{3}-(7 / 6+t / 6) z^{2}+\frac{5 t}{24} z+\frac{t}{48}=0
$$

By the root formula of a cubic equation(see [24]), its second root is given by

$$
\frac{1-\hat{l}_{2}(\kappa)}{2}=-\iota \cos (\psi+\pi / 3)+\frac{7+t}{18},
$$

where $\iota=2\left(-\frac{w}{3}\right)^{1 / 2}, \cos 3 \psi=-\frac{q}{2}\left(-\frac{w}{3}\right)^{-3 / 2}, w=-\frac{49}{108}+\frac{17 t}{216}-\frac{t^{2}}{108}, q=-\frac{343}{2916}+$ $\frac{25 t}{486}+\frac{17 t^{2}}{3888}-\frac{t^{3}}{2916}$. Hence, by the Taylor expansion, one has

$$
\frac{1-\hat{l}_{2}(\kappa)}{2}=\frac{t^{1 / 2}}{2 \sqrt{14}}+O(t)=\frac{\kappa^{-1 / 2}}{2 \sqrt{14}}+O\left(\kappa^{-1}\right) .
$$

Note that $\hat{N}(\kappa)=\left[\frac{\pi}{4 \arcsin \sqrt{\frac{1-\hat{l}(\kappa)}{2}}}\right]+1$, the proof is completed by (3.18) and (3.19). 
Lemma 3.6 Let $\varrho>0$ be such that the smooth cavity solution $r(\cdot)$ is well defined on $[\varrho, 1]$ and satisfies $r(R) \geq r_{c}>0,(2.7)$ and $\left|r^{(3)}(R)\right| \leq Q$. Then, there exists a constant $C>0$, such that (3.13) holds, if $\epsilon \geq \max \left\{\varrho, C \tau^{2}\right\}$ and $\epsilon+\tau \leq 1$. Furthermore, let $\tilde{\tau}_{0}=\min \left\{1-\epsilon, \sqrt{\frac{2 r_{c}}{\max _{\xi \in[\varrho, 1]} r^{\prime \prime}(\xi)}}\right\}$, then (3.1) holds for all $\tau \in$ $\left(0, \tilde{\tau}_{0}\right]$.

Proof. Taylor expanding $r\left(\epsilon+\frac{\tau}{2}\right), r(\epsilon+\tau)$ at $\epsilon$, one gets

$$
4 r_{1 / 2}-3 r_{0}-r_{1}=r^{\prime}(\epsilon) \tau+\frac{1}{12} r^{(3)}\left(\xi_{1}\right) \tau^{3}-\frac{1}{6} r^{(3)}\left(\xi_{2}\right) \tau^{3}
$$

Since $r^{\prime}(\epsilon) \geq m \epsilon$ by $(2.7)$, (3.13) follows by taking $C=\frac{1}{2 m} Q$.

On the other hand, by the Taylor expansion and $r^{\prime \prime}(x)>0$, we have

$$
2 r_{1 / 2}-r_{1}>r(\epsilon)-r^{\prime \prime}(\eta) \frac{\tau^{2}}{2}, \text { for some } \eta \in(\epsilon, \epsilon+\tau)
$$

This yields the inequality (3.1).

The orientation-preservation conditions on the mesh can now be given as follows, where, to simplify the notations, we set $\kappa_{0} \triangleq \kappa_{0}^{r}=\frac{r(\epsilon)}{r(\epsilon+\tau)}, \kappa_{1 / 2} \triangleq \kappa_{1 / 2}^{r}=\frac{r\left(\epsilon+\frac{\tau}{2}\right)}{r(\epsilon+\tau)}$.

Theorem 3.7 Let $u(x)=\frac{r(|x|)}{|x|} x$ be the cavity solution satisfying the conditions of Lemma 3.6, and $\Pi u(x)$ be the interpolation function of $u(x)$ on a quadratic iso-parametric finite element function space defined on a mesh consisting of only elements of types $A$ and $B$. Then, there exist constants $\tilde{\tau}, C>0$, and an integer $\tilde{N}\left(\kappa, \kappa_{0}, \kappa_{1 / 2}\right)$, such that det $\frac{\partial \Pi u}{\partial x}>0$ on each of the finite elements, if the mesh satisfies the conditions that $\tau \leq \tilde{\tau}, \epsilon \geq C \tau^{2}$ and $N \geq \tilde{N}\left(\kappa, \kappa_{0}, \kappa_{1 / 2}\right)$. Moreover, $\tilde{N}^{-1}\left(\kappa, \kappa_{0}, \kappa_{1 / 2}\right) \sim(\epsilon \tau)^{\frac{1}{4}}$.

Proof. Since $u(x)=\frac{r(|x|)}{|x|} x$ is the smooth minimizer of (2.3), it follows from Lemma 2.1 that Lemma 3.6 holds for $r(R)$. Taking $v(x)=u(x)$, or equivalently $s(t)=r(t)$, in Lemma 3.1, let $\tilde{\tau}, C$ be given by Lemma 3.6, then it follows from Theorem 3.4 and Corollary 3.5 that, on a mesh subject to the constraints $\tau \leq \tilde{\tau}$ and $\epsilon \geq C \tau^{2}$, the interpolation function $\Pi u$ is orientation preserving, i.e., $\operatorname{det} \frac{\partial \Pi u}{\partial x}>0$ if and only if $N \geq \tilde{N} \triangleq \max \left\{\hat{N},\left[\frac{\pi}{2 \arccos l}\right]+1\right\}$, where $l=\max \left\{l_{1}, l_{2}\right\}$ with $l_{1}$, 
$l_{2}$ being given in Theorem 3.4 by setting $s(t)=r(t)$. What remains to show is $\tilde{N}^{-1}\left(\kappa, \kappa_{0}, \kappa_{1 / 2}\right) \sim(\epsilon \tau)^{\frac{1}{4}}$.

Note that

$$
\begin{aligned}
1-l_{1} & =\frac{\sqrt{r_{1 / 2}^{2}-r_{0}^{2} / 2-r_{0} r_{1} / 2}+r_{0}-r_{1 / 2}}{r_{0}} \\
& =\frac{\sqrt{\frac{r^{\prime}(\epsilon)}{2} \tau+\frac{r^{(3)}\left(\xi_{1}\right)}{24} \tau^{3}-\frac{r^{(3)}\left(\xi_{2}\right)}{12} \tau^{3}+O\left(\epsilon^{2} \tau^{2}+\epsilon \tau^{3}+\tau^{4}\right)}-O\left(\epsilon \tau+\tau^{2}\right)}{\sqrt{r_{0}}},
\end{aligned}
$$

where $\xi_{1} \in(\epsilon, \epsilon+\tau / 2), \xi_{2} \in(\epsilon, \epsilon+\tau)$. Since $m \epsilon \leq r^{\prime}(\epsilon) \leq M \epsilon, \epsilon \geq C \tau^{2}$, then $1-l_{1} \preceq(\epsilon \tau)^{1 / 2}$. On the other hand, by taking $C=\frac{Q}{2 m}$ as in Lemma 3.6, one has that $\frac{r^{\prime}(\epsilon)}{2} \tau+\frac{r^{(3)}\left(\xi_{1}\right)}{24} \tau^{3}-\frac{r^{(3)}\left(\xi_{2}\right)}{12} \tau^{3} \geq \frac{m \epsilon \tau}{2}-\frac{Q}{8} \tau^{3} \geq \frac{m}{4} \epsilon \tau$. Thus $1-l_{1} \sim(\epsilon \tau)^{1 / 2}$. Denote $\tilde{l}_{2}=\frac{1}{2}\left(1-l_{2}\right)$, then $\tilde{l}_{2}$ is the second root of the equation (see $\left.(3.15)\right)$

$$
-6(1-2 z)^{3}+4 \kappa_{1 / 2}(1-2 z)^{2}+\left(\kappa_{0}+9\right)(1-2 z)-8 \kappa_{1 / 2}=0 .
$$

By the root formula of a cubic equation, $\tilde{l}_{2}$ is given by

$$
\tilde{l}_{2}=-\iota \cos \left(\psi+\frac{\pi}{3}\right)-\frac{\kappa_{1 / 2}}{9}+\frac{1}{2}
$$

where $\iota=2\left(-\frac{w}{3}\right)^{1 / 2}, \cos 3 \psi=-\frac{q}{2}\left(-\frac{w}{3}\right)^{3 / 2}, w=-\frac{3}{8}-\frac{\kappa_{0}}{24}-\frac{\kappa_{1 / 2}^{2}}{27}, q=-\frac{\kappa_{1 / 2}}{8}+\frac{\kappa_{0} \kappa_{1 / 2}}{216}+$ $\frac{2 \kappa_{1 / 2}^{3}}{729}$. By the Taylor expansion, we obtain

$$
\begin{gathered}
\iota=\frac{7}{9}\left(1-\frac{17}{196} \frac{r^{\prime}(\epsilon+\tau)}{r_{1}} \tau+\frac{13 r^{\prime \prime}(\epsilon+\tau) \tau^{2}}{392 r_{1}}\right)+O\left(\epsilon^{2} \tau^{2}+\tau^{3}\right), \\
\cos 3 \psi=1-\frac{243 r^{\prime}(\epsilon+\tau) \tau}{1372 r_{1}}+O\left(\epsilon^{2} \tau^{2}+\tau^{3}\right) .
\end{gathered}
$$

This leads to

$$
\sin 3 \psi=\frac{9}{7} \sqrt{\frac{3 r^{\prime}(\epsilon+\tau) \tau}{14 r_{1}}}\left(1+O\left(\epsilon^{2}+\tau\right)\right),
$$

and

$$
\psi=\frac{3}{7} \sqrt{\frac{3 r^{\prime}(\epsilon+\tau) \tau}{14 r_{1}}}\left(1+O\left(\epsilon^{2}+\tau\right)\right) .
$$


Consequently, we get

$$
\begin{aligned}
\tilde{l}_{2} & =-\frac{\iota}{2} \cos \psi+\frac{\sqrt{3}}{2} \iota \sin \psi-\frac{\kappa_{1 / 2}}{9}+\frac{1}{2} \\
& =-\frac{\iota}{2}+\frac{\sqrt{3}}{2} \iota \psi-\frac{\kappa_{1 / 2}}{9}+\frac{1}{2}+O\left(\psi^{2}\right) \\
& =\frac{1}{2} \sqrt{\frac{r^{\prime}(\epsilon+\tau) \tau}{14 r_{1}}}+O\left(\epsilon \tau+\tau^{2}\right), \\
& \sim \sqrt{\epsilon \tau+\tau^{2}} .
\end{aligned}
$$

Hence, $1-l_{2} \sim((\epsilon+\tau) \tau)^{1 / 2}$. The conclusion of the theorem now follows by the definition of $\tilde{N}$, Corollary 3.5 and $\arccos l=2 \arcsin \sqrt{\frac{1-l}{2}}$.

Remark 3.8 We would like to point out that the condition $\epsilon \geq C \tau^{2}$ on the mesh in Theorem 3.7 is not necessary. It is just a sufficient condition to ensure (3.13). For an incompressible cavity solution, (3.13) is in fact unconditionally satisfied, and thus no restriction on the thickness $\tau$ is required. Of course this does not change the fact that the quadratic iso-parametric element on its own is unstable for incompressible elasticity, however the result could be useful for a properly coupled mixed finite element method. In the proof above, we could as well obtain $\tilde{N}^{-1} \preceq$ $\left(\epsilon \tau+\tau^{3}\right)^{1 / 4}$ without the condition $\tau \preceq \epsilon^{1 / 2}$.

Remark 3.9 For the nonsymmetric cavitation deformation, under certain regularity assumptions on the solution, we can apply similar methods as in [22] to obtain a sufficient condition for the interpolation function to be orientation preserving.

Remark 3.10 Compared to the orientation-preservation condition for the dualparametric bi-quadratic FEM in [22], where the corresponding sufficient and necessary condition for the interpolation of the radially symmetric cavity solution is (3.13) only, while the quadratic iso-parametric FEM imposes additional restrictions on the mesh distribution in the angular direction, which can be more severe a condition. However, when the radius @ of the initial defect is very small, to achieve the optimal interpolation error, similar restrictions on the mesh distribution in the 


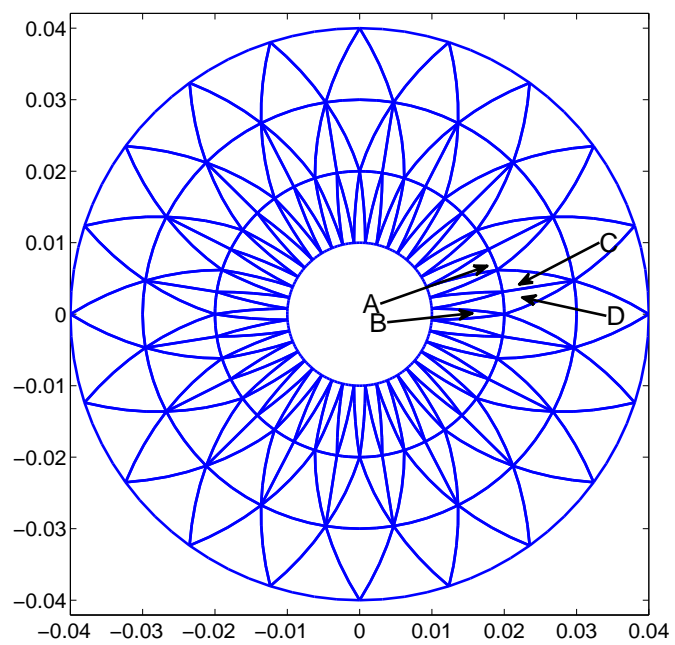

Figure 7: Mesh coarsening is easily achieved.

angular direction are also required for the dual-parametric bi-quadratic FEM, particularly in the non-radially-symmetric case [21, 22]. Thus, to control the total degrees of freedom of the mesh, it is often necessary, for both triangular and rectangular triangulations, to coarsening the mesh layers away from the cavity. For our curved triangular partition, a conforming finite element mesh coarsening from a circular ring layer to the next one outside can be easily achieved, by dividing each type $B$ element in the outside layer into two (types $C$ and $D$ as shown in Figure 7 ) with a straight line right in the middle along the radial direction without deteriorating the orientation preservation and the approximation property, while it can be hardly done for the curved rectangular one without introducing an intermediate layer. Hence, for a conforming finite element cavity approximation, the quadratic iso-parametric FEM can still be advantageous.

As a comparison, we present below the orientation-preservation condition for the conforming affine element.

Theorem 3.11 For the cavitation solution $u(x)=\frac{r(|x|)}{|x|} x$, the interpolation function in the conforming affine finite element space is orientation preserving if and only if $N \geq \tilde{N}_{a}$, where $((\epsilon+\tau) \tau)^{-1 / 2} \preceq \tilde{N}_{a} \preceq(\epsilon \tau)^{-1 / 2}$. 
Proof. For the radially symmetric deformation $v(x)=\frac{s(|x|)}{|x|} x$, the interpolation function in the affine finite element space is given by $\Pi v(x)=\sum_{i=1}^{3} b_{i} \hat{\lambda}_{i}(\hat{x})$. As in Figure 1(a), we can work on a typical triangle with $b_{1}=\left(0, s_{0}\right), b_{2}=s_{1}\left(\sin \frac{\pi}{N}, \cos \frac{\pi}{N}\right)$ and $b_{3}=s_{1}\left(-\sin \frac{\pi}{N}, \cos \frac{\pi}{N}\right)$. Thus

$$
\Pi v(x)=\left(s_{1} \sin \frac{\pi}{N}\left(\hat{x}_{1}-\hat{x}_{2}\right), s_{0}\left(1-\hat{x}_{1}-\hat{x}_{2}\right)+s_{1} \cos \frac{\pi}{N}\left(\hat{x}_{1}+\hat{x}_{2}\right)\right),
$$

$\operatorname{det} \frac{\partial \Pi v}{\partial \hat{x}}=2 s_{1} \sin \frac{\pi}{N}\left(s_{1} \cos \frac{\pi}{N}-s_{0}\right)$. Hence the mesh is well defined, i.e., $\operatorname{det} \frac{\partial x}{\partial \hat{x}}>0$ if and only if $\cos \frac{\pi}{N}>\frac{\epsilon}{\epsilon+\tau}$. It follows that for $u(x)=\frac{r(|x|)}{|x|} x$, det $\frac{\partial \Pi u}{\partial x}>0$ if and only if $N>\tilde{N}_{a} \triangleq \max \left\{\frac{\pi}{\arccos \frac{r(\epsilon)}{r(\epsilon+\tau)}}, \frac{\pi}{\arccos \frac{\epsilon}{\epsilon+\tau}}\right\}$. The conclusion is then established by $(2.7)$.

In [25], it is shown that a necessary condition for the conforming piecewise affine finite element interpolation function of a cavity solution to have finite energy in the layer $\Omega_{(\epsilon, \tau)}$ is $\tau \preceq \epsilon^{p-1}$, where $p$ is the parameter in the energy density function (2.1). For $p=3 / 2$, this coincides with the condition $\tau \preceq \epsilon^{1 / 2}$ used in Theorem 3.7. It is interesting to see that, on a circular ring domain $\Omega_{(\epsilon, \tau)}$, by Theorems 3.7 and 3.11, $\tilde{N} \sim \tilde{N}_{a}^{1 / 2}$ when $\tau \preceq \epsilon$, while $\tilde{N}_{a}^{1 / 2} \preceq \tilde{N} \preceq \tilde{N}_{a}^{3 / 4}$ when $\tau^{2} \preceq \epsilon \preceq \tau$, i.e., the quadratic iso-parametric finite element approximation needs significantly less elements. For $2>p>3 / 2$, when the cavitation solution is harder to obtain numerically, the restriction on the mesh for the conforming piecewise affine FEM is harsher, which means a much larger number of total degrees of freedom is required. The fact, that the number of elements needed on a layer with $\epsilon$ small so much exceeds one's intuitive expectation, partially explains why no successful attempt has ever been made at applying the affine FEM to the cavitation computation.

To illustrate the potential of our analysis in cavitation computation, we present below some numerical results. The energy density in the numerical experiments is given by $(2.1)$ with $p=3 / 2, \omega=2 / 3$, and $g(x)=2^{-1 / 4}\left(\frac{1}{2}(x-1)^{2}+\frac{1}{x}\right)$, the domain is $\Omega_{0.01} \subseteq \mathbb{R}^{2}$ with a displacement boundary condition $u_{0}(x)=2 x$ given on $\Gamma_{0}=\partial B_{1}(0)$ and a traction free boundary condition given on $\Gamma_{1}=\{x:|x|=0.01\}$.

Figure 8 compares the $L^{2}$ error of the finite element cavity solutions $u_{h}$ against the total degrees of freedom $N_{s}$, where our result is obtained on the meshes produced according to our analytical results (near the cavity it is essentially governed 
by the orientation-preservation condition, see also [21]), while the meshes used in [11] were provided according to limited numerical experiences and thus, to guarantee the orientation preservation, the thickness of the circular annulus were taken much thicker than necessary in general. It is clearly seen that our mesh is better in convergence rate as well as actual accuracy.

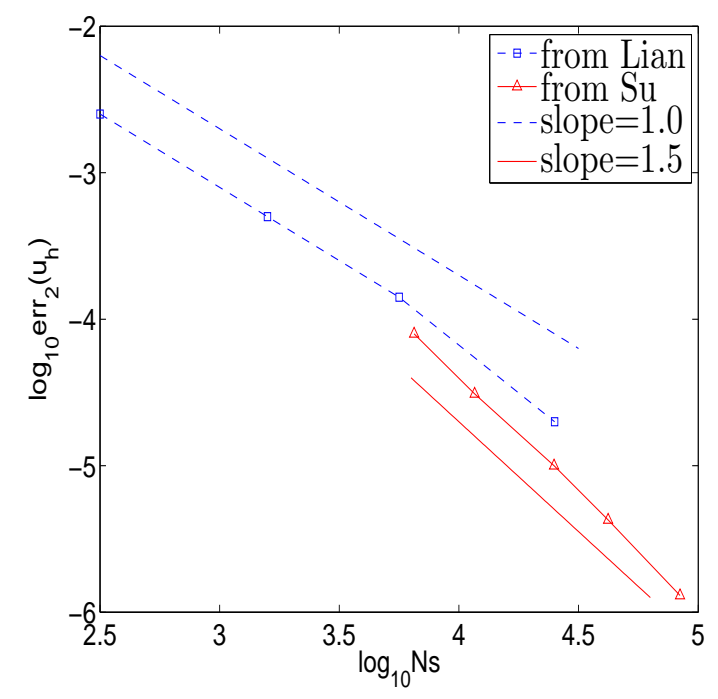

Figure 8: $L^{2}$ error of numerical cavity solutions obtained on meshes based on experiences and a priori analysis.

\section{Conclusion remarks and discussions}

The orientation-preservation condition, i.e., the Jacobian determinant of the deformation gradient $\operatorname{det} \nabla u>0$, is a natural physical constraint in elasticity as well as in many other fields. It is well known that the constraint can often cause serious difficulties in both theoretical analysis and numerical computation, especially when the material is subject to large deformation as in the case of cavitation. To overcome such difficulties can be crucial to successfully solve the related problems.

In this paper, we analyzed the quadratic iso-parametric finite element interpolation functions of the radially symmetric cavitation deformation on a class of large radially symmetric expansion accommodating meshes, and obtained a set of 
sufficient and necessary conditions on the orientation-preservation, which provide a practical quantitative guide for the mesh distribution in the neighborhood of a cavity in both radial and angular directions. Furthermore, the result shows that the orientation-preserving cavitation approximation can be achieved by the quadratic iso-parametric finite element method with a reasonable number of total degrees of freedom, which is significantly smaller than the conforming piecewise affine finite element method and is somehow comparable to the bi-quadratic dual-parametric finite element method [22]. In fact, the orientation-preservation conditions together with the interpolation error estimates, which will be established in a separate paper of ours [21], will allow us to establish, for the quadratic iso-parametric FEM, a meshing strategy leading to numerical cavitation solutions with optimal error bounds comparable to the ones obtained in [22] for a dual-parametric bi-quadratic FEM.

\section{References}

[1] Bai, Y., Li, Z., Numerical solution of nonlinear elasticity problems with Lavrentiev phenomenon. Math. Models Methods Appl. Sci., 17 (2007), 1619-1640.

[2] Ball, J. M., Discontinuous equilibrium solutions and cavitation in nonlinear elasticity. Philos. Trans. R. Soc. London, A 306 (1982), 557-611.

[3] Ball, J. M., A version of the fundamental theorem for Young measures. Lecture Notes in Physics, 344 (1989), 207-215.

[4] Ball, J. M., Knowles, G., A numerical method for detecting singular minimizers. Numer. Math., 51 (1987), 181-197.

[5] Chi, H., Talischi, C., Lopez-Pamies, O., H.Paulino, G., Polygonal finite elements for finite elasticity. Int. J. Numer. Meth. Engng., 101 (2015), 305-328. 
[6] Gent, A. N., Lindley, P. B., Internal rupture of bonded rubber cylinders in tension. Proc. R. Soc. London, A 249 (1958), 195-205.

[7] Henao, D., Cavitation, invertibility, and convergence of regularized minimizers in nonlinear elasticity. J. Elast., 94 (2009), 55-68.

[8] Lavrentiev, M., Sur quelques problems du calcul des variations. Ann. Math. Pure Appl., 4 (1926), 7-28.

[9] Li, Z., A numerical method for computing singular minimizers. Numer. Math., 71 (1995), 317-330.

[10] Lian, Y., Li, Z., A dual-parametric finite element method for cavitation in nonlinear elasticity. J. Comput. Appl. Math., 236 (2011), 834-842.

[11] Lian, Y., Li, Z., A numerical study on cavitations in nonlinear elasticity-defects and configurational forces. Math. Models Methods Appl. Sci., 21 (2011), 2551-2574.

[12] Lian, Y., Li, Z., Position and size effects on voids growth in nonlinear elasticity. Int. J. Fracture, 173 (2012), 147-161.

[13] Lopez-Pamies, O., Idiart, M. I., Nakamura, T., Cavitation in elastomeric solids: I-A defect-growth theory. J. Mech. Physics Solids, 59 (2011), 1464-1487.

[14] Negrón-Marrero, P. V., Betancourt, O., The numerical computation of singular minimizers in two-dimensional elasticity. J. Comput. Phys., 113 (1994), 291-303.

[15] Müller, S., Spector S. J., An existence theory for nonlinear elasticity that allows for cavitation. Arch. Ration. Mech. Anal., 131 (1995), 1-66.

[16] Müller, S., Variational models for microstructure and phase transitions. Lecture Notes in Math., 1713 (1999), 85-210. 
[17] Sivaloganathan, J., Uniqueness of regular and singular equilibia for spherically symmetric problems of nonlinear elasticity, Arch. Ration. Mech. Anal., 96 (1986), 97-136.

[18] Sivaloganathan, J., Spector. S. J., Tilakraj. V., The convergence of regularized minimizers for cavitation problems in nonlinear elasticity. SIAM J. Appl. Math., 66 (2006), 736-757.

[19] Sivaloganathan, J., Negro-Marrero, P. V., The Numerical computation of the critical boundary displacement for radial cavitation. Math. Mech. Solids, 14 (2009), 696-726.

[20] Sivaloganathan, J., Spector, S. J., On cavitation, configurational forces and implications for fracture in a nonlinearly elastic material. J. Elast., 67 (2002), $25-49$.

[21] Su, C., Li, Z., A meshing strategy for a quadratic iso-parametric FEM in cavitation computation in nonlinear elasticity. Preprint.

[22] Su, C., Li, Z., Error Analysis of a Dual-parametric Bi-quadratic FEM in Cavitation Computation in Elasticity. SIAM J. Numer. Anal., 53(3), (2015), 1629-1649.

[23] Tvergaard V., On cavitation instabilities with interacting voids. Euro. J. Mech. - A/Solids, 32, (2012), 52-58.

[24] Wang Z., Guo D., Introduction to Special Functions, Peking University Press, Beijing, 2006.

[25] Xu, X., Henao, D., An efficient numerical method for cavitation in nonlinear elasticity. Math. Models Methods Appl. Sci., 21 (2011), 1733-1760. 